\title{
Different mental representations for place recognition and goal localization
}

\author{
Christine Valiquette and Timothy P. MCNamara \\ Vanderbilt University, Nashville, Tennessee
}

\begin{abstract}
Determining one's current location and locating a goal relative to one's position are important components of successful human navigation in familiar environments. Several prominent cognitive theories of human spatial memory (e.g., McNamara, 2003; Sholl, 2001; Wang \& Spelke, 2002) assume that both behaviors access the same enduring mental representations of the environment. Participants in the present experiment learned the locations of seven objects within a room from two views, and were then tested in a separate room using scene recognition and judgments of relative direction (JRD). Scene recognition results indicated that two viewercentered representations of the layout of objects were preserved in long-term memory, whereas JRD showed evidence of a single orientation-dependent long-term mental representation. The challenges of incorporating the present findings into existing theories of human spatial memory are discussed.
\end{abstract}

Successful human navigation in familiar environments often depends on two component processes: determining one's location and locating a goal in relation to one's position. Determining one's current location is essential for staying oriented in, and reorienting with respect to, the environment and may depend on the ability to recognize previously experienced configurations of objects or landmarks (e.g., recognizing an intersection of two streets based on a view of the buildings that surround it). Determining the location of a goal in relation to one's present position is important for navigation in large scale space and finding novel ways of reaching unseen goals (e.g., finding a novel detour in order to avoid a high traffic area at rush hour). Following a route in a familiar environment can be construed as alternating between determining one's present location and determining the location of a goal, with the goal being the next landmark on the route.

Several prominent cognitive theories of human spatial memory assume, at least implicitly, that the same enduring mental representations are used to recognize one's present position and to determine the location of goals in relation to one's present position. ${ }^{1}$ According to Wang and Spelke's $(2000,2002)$ theory, long-term spatial memory relies on a viewpoint dependent representational system and a geometry-based reorientation system. The viewpoint dependent system stores "viewer-centered" (Wang $\&$ Spelke, 2002) representations of landmarks and scenes. The geometric shape of the environment (e.g., the shape of a room), but not spatial relations among objects in the environment, is represented in an enduring allocentric system. Place recognition is accomplished by the viewpoint dependent system. It is not clear in this model how locating a goal location would be accomplished but this process would also seem to depend on the viewpoint dependent system as this is the only system in the model that stores long-term representations of the locations objects and landmarks.

The theory proposed by Mou, McNamara, and their colleagues (Mou \& McNamara, 2002; Mou, McNamara, Valiquette, \& Rump, 2004; Shelton \& McNamara, 2001a) stipulates that long-term spatial memory is supported by an environmental subsystem, which represents enduring features of the environment. This subsystem uses intrinsic reference systems (e.g., rows and columns formed by chairs in a classroom) to represent object-to-object spatial relations in orientation dependent representations. Although the nature of the processes involved has not been specified in detail, both recognition of one's current location and the determination of the location of a goal are assumed to depend on spatial information stored in the environmental subsystem.

Finally, in Sholl's model (Easton \& Sholl, 1995; Sholl, 2001; Sholl \& Nolin, 1997), spatial relations among objects are represented in an object-to-object system using an orientation-independent reference system. Our interpretation of this model is that place recognition and locating distant goals would be accomplished in the object-toobject system. ${ }^{2}$

The results of several recent experiments indicate that recognizing a configuration of objects and determining where a goal is in relation to a position within that configuration may rely upon different types of spatial information (Shelton \& McNamara, 2001b, 2004a, 2004b). In these experiments, different patterns of performance were observed in scene recognition, which involves recognizing configurations of collections of objects, and in judgments 


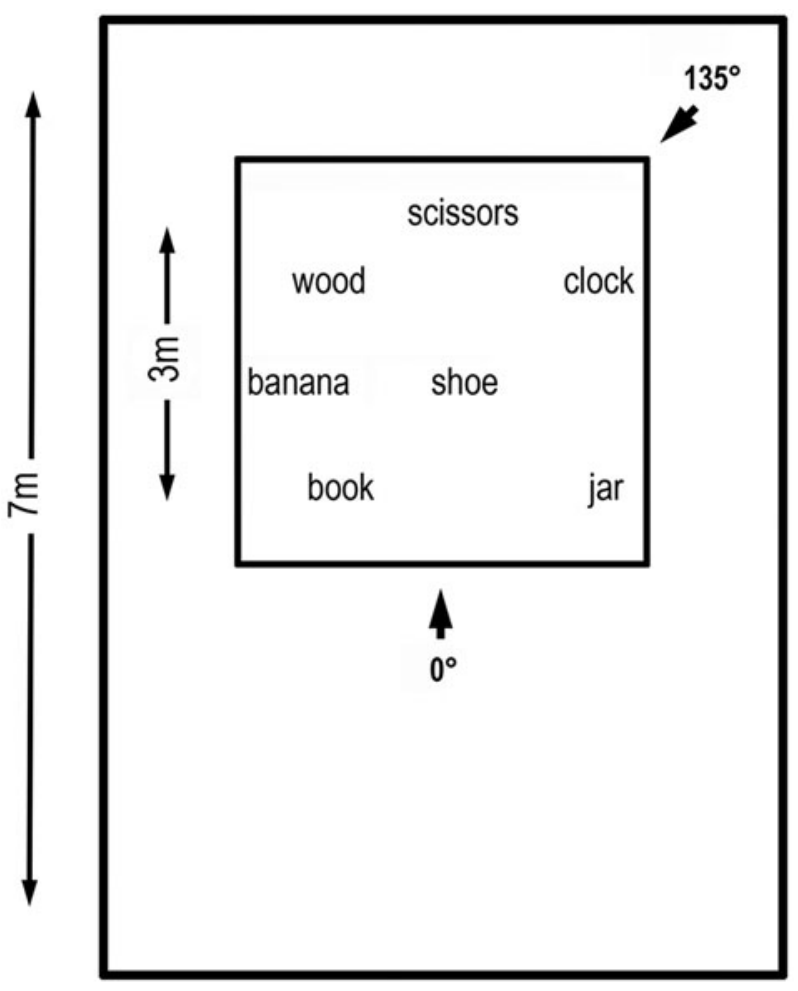

$5 \mathrm{~m}$

Figure 1. A diagram of one of the study configurations. The aligned $\left(0^{\circ}\right)$ and the misaligned $\left(135^{\circ}\right)$ viewing positions, the dimensions of the mat, and the dimensions of the room are indicated.

of relative direction (JRD), which involves judging the direction of a target object relative to an imagined position. Possible limitations of these experiments are that different perspectives were experienced in different modalities (e.g., visual vs. tactile) or only via virtual reality.

The experiment reported in this article explored the possibility that the task being performed determines the type of memory accessed to perform it by comparing JRD and scene recognition performance when the amount and quality of experience at each study view are equivalent. The experiment was based on a paradigm developed by Shelton and McNamara (2001a). Participants learned a room-sized layout of objects by studying it from two views, one aligned, and one misaligned, with the walls of the study room and the edges of a mat on which the objects were placed (see Figure 1). Their memories for the layout were then probed using both scene recognition and JRD.

Each of the three theories discussed earlier predicts different patterns of results. Wang and Spelke's theory predicts that the two experienced views will show a benefit over novel views, as experienced views should be stored in the viewpoint dependent system. Mou, McNamara, and colleagues' theory predicts that only the experienced view aligned with the intrinsic frame of reference of the layout, made salient by its alignment with the walls of the room and edges of the mat, will show a benefit over novel views.
Sholl's model predicts that performance will be the same for studied and novel views (assuming that the learning conditions are sufficient to produce an orientation independent representation). None of these theories contains representations or processes that would allow it to predict different patterns of performance for scene recognition and JRD.

\section{METHOD}

\section{Participants}

Forty-eight undergraduates participated for course credit. The JRD data from one participant were eliminated because her average error was above $65^{\circ}$ (chance performance $=90^{\circ}$ ) and more than $10^{\circ}$ higher than the next highest individual average error.

\section{Materials and Design}

Two configurations of seven objects were used (one is illustrated in Figure 1). The same seven objects, and the same seven object locations, were used in both configurations. The difference between configurations was the assignment of objects to locations which was determined randomly.

Each scene recognition test trial required participants to determine if a picture presented on a computer screen was of the configuration of objects that they had studied or a picture of the same objects occupying different locations (i.e., the other layout). The primary independent variable was pictured heading. Eight equally spaced headings were used. Headings were identified counterclockwise from $0^{\circ}$ to $315^{\circ}$ in $45^{\circ}$ steps (see Figure 1). Participants received a total of 160 trials, comprised of 10 blocks of 16 randomly presented trials, one trial at each target pictured heading (a picture of the studied layout) and one trial at each distractor pictured heading (a picture of the other layout), with a break between blocks five and six.

The principal dependent measure was response latency on trials in which the target (studied) layout was pictured. Error data were also collected.

Each JRD test trial was constructed from the names of three objects in the room and required participants to point to an object as if standing in a particular position within the room; for example, "Imagine you are standing at the jar and facing the shoe; point to the clock." The primary independent variable was imagined heading. Headings were identified counterclockwise from $0^{\circ}$ to $315^{\circ}$ in $45^{\circ}$ steps. Pointing direction, defined as the direction of the target object relative to the imagined heading, was varied systematically by dividing the space about a given heading into three regions: Front $\left(0^{\circ}-60^{\circ}\right.$ and $\left.300^{\circ}-360^{\circ}\right)$, sides $\left(60^{\circ}-120^{\circ}\right.$ and $\left.240^{\circ}-300^{\circ}\right)$, and back $\left(120^{\circ}-240^{\circ}\right)$. Pointing direction was approximately counterbalanced across imagined headings. Participants received a total of 72 trials, nine at each of the eight imagined headings.

The principal dependent measure was the angular error of the pointing response, measured as the absolute angular difference between the judged pointing direction and the actual direction of the target. Response latencies were also collected.

\section{Procedure}

Twenty-four (12 female) participants learned each layout. Order of viewing $\left(0^{\circ}-135^{\circ}, 135^{\circ}-0^{\circ}\right)$ was counterbalanced across layout and gender. Before entering the study room, each participant was instructed to learn the objects and their locations for a spatial memory test. The participant was blindfolded and led into the study room to the first viewing position. The blindfold was removed, and the experimenter named the objects while pointing to them in a random order. The participant then viewed the display for 30 seconds before being asked to close his or her eyes. The experimenter then named the objects in a random order, as the participant pointed to them. The first study phase was complete when the participant correctly pointed to each of the objects on two consecutive trials, and then, after studying 


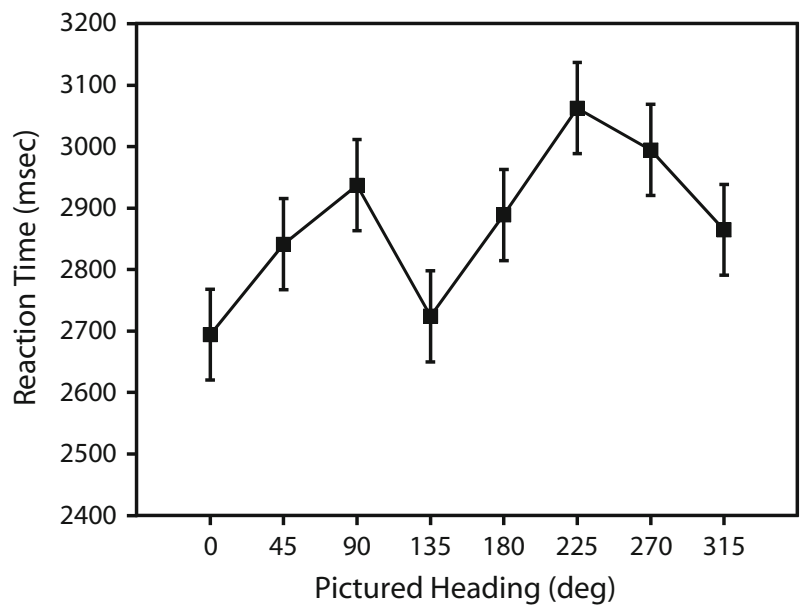

Figure 2. Reaction time on scene recognition target trials as a function of pictured heading. Error bars are confidence intervals corresponding to \pm 1 standard error of the mean as estimated from the analysis of variance.

the layout the last time, correctly named and pointed to each of the objects with his or her eyes closed. The participant was then blindfolded and led to the second viewing position, and the learning process was repeated. When the participant had successfully learned the objects' locations, the blindfold was replaced and the participant was led from the room. Testing took place on a different floor of the building.

The scene recognition test trials were presented on a Macintosh computer, using SuperLab Pro 1.75. Twenty-four (12 female) participants saw pictures of the target and distractor layouts that had been modified using Adobe PhotoShop in order to remove the walls of the room. The other twenty-four (12 female) participants saw pictures that included the walls of the room. This manipulation was included to determine if the frame of reference provided by the walls of the room would affect scene recognition performance. Participants were instructed to press the " $\mathrm{J}$ " key if the picture presented was of the layout that they had studied and to press the "F" key if it was not. The experimenter remained in the room for the first four trials to answer any questions. Trials performed while the experimenter was present were removed from the data prior to analysis.

The JRD test trials were presented on a Macintosh computer. Participants first received four practice trials involving locations on campus. Each test trial was initiated by the participant and proceeded as follows: The statement specifying the imagined vantage point and target object was displayed (e.g., "Imagine you are standing at the jar, facing the shoe. Point to the clock."), along with a simulated dial and pointer (see Shelton \& McNamara, 1997). The participant used the mouse to position the pointer on the dial to indicate the direction of the target object relative to the imagined heading, which corresponded to "up" in the display. The participant clicked the mouse to finish the response. Participants were told that it was "more important to be accurate than fast."

\section{RESULTS}

Mean reaction time in the scene recognition task, collapsed across participants, and with error trials removed, is plotted in Figure 2 as a function of pictured heading. Responses were faster for the pictured headings of $0^{\circ}$ and $135^{\circ}$, which corresponded to the orientations of the study views, than for all other headings.

Mean reaction time was computed for each participant and each condition, and analyzed in a split-plot ANOVA with terms for layout, viewing order $\left(0^{\circ}\right.$ first or $135^{\circ}$ first $)$, gender, the presence of walls in the pictures, and pictured heading $\left(0^{\circ}-315^{\circ}\right.$ in $45^{\circ}$ increments $)$. Pictured heading was within-participants.

The main effect of pictured heading was significant $\left[F(7,308)=2.74, M S_{\mathrm{e}}=263,615.88, p<.01\right]$. Pairwise comparisons showed that performance at $0^{\circ}$ and $135^{\circ}$ was significantly better than performance at all other headings $[t \mathrm{~s}(308) \geq 1.96]$. The interactions between viewing order and heading and between presence of walls and heading were not significant ( $p s>.30)$. No other main effects or interactions reached significance. ${ }^{3}$

The average error on target trials across participants was very low at $1.65 \%$, with participants missing between zero and five (of 80) trials each. There was no evidence of a speed-accuracy trade-off.

As shown in Figure 3, the pattern of results in JRD was different from that in scene recognition. Mean absolute angular error in JRD was computed for each participant and each condition, and analyzed in a split-plot ANOVA with terms for order of viewing $\left(0^{\circ}-135^{\circ}\right.$ or $\left.135^{\circ}-0^{\circ}\right)$, layout, gender, imagined heading $\left(0^{\circ}-315^{\circ}\right.$ in $45^{\circ}$ increments $)$, and pointing direction (front, sides, back). Imagined heading and pointing direction were within-participants. Reaction time data were analyzed in the same way.

The main effect of imagined heading on angular error was significant $\left[F(7,301)=16.36, M S_{\mathrm{e}}=353.96, p<\right.$ .0001]. Participants were more accurate for the imagined heading of $0^{\circ}$ than for all other imagined headings, including $135^{\circ}[t \mathrm{~s}(301) \geq 3.50]$. Performance for $135^{\circ}$ was numerically less accurate than performance for four novel imagined headings $\left(45^{\circ}, 90^{\circ}, 180^{\circ}, 270^{\circ}\right)$, although only one of these comparisons was statistically significant $\left(135^{\circ}\right.$ vs. $\left.90^{\circ}\right)[t(301)=3.62]$. A contrast also showed that, excluding the heading of $0^{\circ}$, pointing accuracy was higher for headings aligned with the walls of the room $\left(90^{\circ}, 180^{\circ}\right.$, and $\left.270^{\circ}\right)$ than for misaligned headings $\left(45^{\circ}\right.$, $135^{\circ}, 225^{\circ}$, and $\left.315^{\circ}\right)\left[F(1,147)=8.85, M S_{\mathrm{e}}=353.96\right.$, $p<.01]$, producing a sawtooth pattern. The pattern in

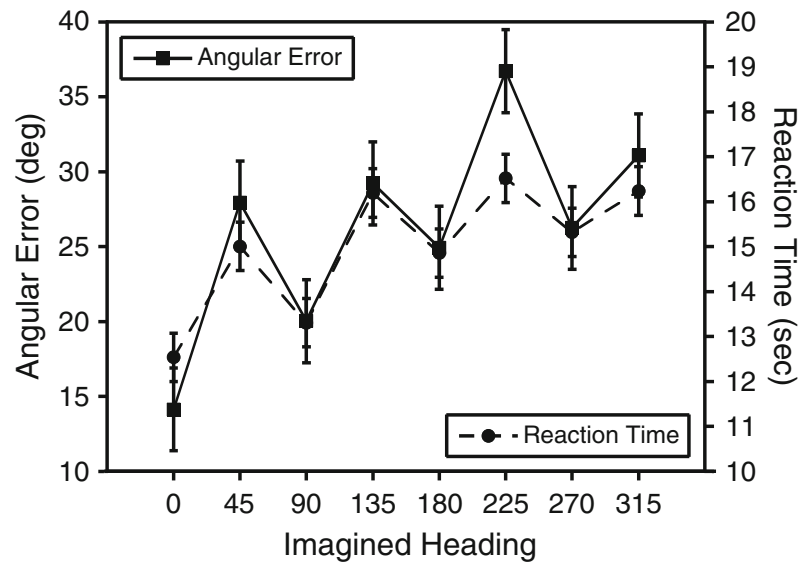

Figure 3. Angular error and reaction time in judgments of relative direction as a function of imagined heading. Error bars are confidence intervals corresponding to \pm 1 standard error of the mean as estimated from the analysis of variance. 
Figure 3 occurred regardless of which view $\left(0^{\circ}\right.$ or $\left.135^{\circ}\right)$ was learned first $(p>.5$ for the viewing order by heading interaction).

The main effect of pointing direction on angular error was significant $\left[F(2,86)=35.70, M S_{\mathrm{e}}=32,426.49\right.$, $p<.0001$ ]. Pointing to the front was more accurate than pointing to the sides, which was more accurate than to the back. The interaction between heading and pointing direction was also significant $\left[F(14,602)=2.98, M S_{\mathrm{e}}=\right.$ $169,969.84, p<.001]$. For pointing judgments to the sides, error was lower for headings of $0^{\circ}, 90^{\circ}, 180^{\circ}$, and $270^{\circ}$ than for other headings, producing a sawtooth pattern. Pointing judgments to the front and to the back did not show this pattern (for an interpretation of this pattern see Valiquette, McNamara \& Smith, 2003). No other interactions or main effects approached significance ( $p s>.1)$.

The reaction time data showed a pattern similar to the angular error data (see Figure 3). There was a main effect of heading $\left[F(7,301)=6.95, M S_{\mathrm{e}}=40,933.89, p<\right.$ .001]. Participants responded more quickly at the imagined heading of $0^{\circ}$ than for all other imagined headings, including $135^{\circ}$ [ts $(301) \geq 3.50$ ], except for $90^{\circ}$. Reaction time for $135^{\circ}$ was numerically longer than reaction time for four novel imagined headings $\left(45^{\circ}, 90^{\circ}, 180^{\circ}, 270^{\circ}\right)$, although only one of these comparisons was statistically significant $\left(135^{\circ} \mathrm{vs} .90^{\circ}\right)[t(301)=3.87]$. The main effect of viewing order approached significance $(p=.09)$. No other main effects or interactions approached significance $(p s>.3)$.

\section{DISCUSSION}

The purpose of the experiment reported in this paper was to determine whether recognizing a location and determining the location of a goal relative to a given position in an environment access different enduring mental representations of the environment. Several prominent theories of human spatial memory and navigation assume, at least implicitly, that these tasks access the same spatial representations in memory. Recent experiments (Shelton \& McNamara, 2001a, 2004a, 2004b), however, have obtained different patterns of results between scene recognition and judgments of relative direction, indicating that place recognition and locating goals may depend on different kinds of information. A possible limitation of those experiments is that different views of the environments were experienced in different modalities (e.g., vision vs. taction \& proprioception) or only via passively viewed movies in desktop VR. The goal of the present experiment was to see if the demands of the memory task determine the type of spatial memory accessed to perform the task, under conditions in which the learning experiences at the two study views were as similar as possible.

The results indicated that performance in JRD and scene recognition tasks relied on different kinds of spatial information. The JRD results suggested that a single orientation-dependent representation of the layout was accessed to perform the task even though two views were experienced. This representation corresponded to the study view that was aligned with salient environmental frames of reference, as predicted by Mou, McNamara, and colleagues' theory (Mou et al., 2004). According to this theory, interobject spatial relations are specified in memory with respect to a small number (typically 1 or 2 ) of intrinsic reference directions (e.g., Mou \& McNamara, 2002). Intrinsic reference directions are selected using egocentric and environmental cues, such that directions emphasized at the time of learning, by direct experience or instructions, are preferred to those which are not, and directions aligned with salient environmental frames of reference are preferred to misaligned directions. Spatial relations that are specified with respect to the dominant reference directions (i.e., those aligned with the walls of the room and edges of the mat in this experiment) can be retrieved from memory, whereas other interobject spatial relations must be inferred (e.g., Klatzky, 1998), creating a cost in terms of accuracy and response time.

The results of the scene recognition task, however, indicated that scene recognition accessed two orientationdependent representations corresponding to the two study views, as predicted by Wang and Spelke's (2000, 2002) theory.

They claim that the human spatial memory and navigation system stores enduring viewer-centered representations of landmarks and scenes. All else equal, experienced views of an environment should be recognized more efficiently than novel views.

The present results can be accommodated in the theory proposed by Mou, McNamara, and colleagues by incorporating enduring viewpoint dependent representations. The challenge for this augmented model is to explain why these representations are apparently not accessed in JRD. One possible explanation is that recall tasks, such as JRD, depend on hippocampal structures and the spatial information recalled to perform JRD is allocentric and represented (at least temporarily) in the hippocampus, whereas recognition tasks depend on cortical regions of the medial temporal lobe and the spatial information accessed for scene recognition is egocentric and represented in those regions of the brain (e.g., Burgess, 2002; Epstein, Graham, \& Downing, 2003; King, Burgess, Hartley, VarghaKhadem, \& O'Keefe, 2002; Mayes et al., 2004; Parslow et al., 2004). Another possible explanation is that scene recognition is a visual task whereas JRD is a spatial task. Ongoing experiments in our laboratory are testing these and other possibilities.

Explaining the present findings in Wang and Spelke's (2000, 2002) theory may be more challenging. Enduring representations of object locations only exist in the viewpoint dependent system. Hence, JRD must access these representations. The challenge is to explain why this task is sensitive to some views (e.g., aligned) but not to others (e.g., misaligned). More generally, it is not obvious how this model can explain results such as Mou and McNamara's (2002), showing that the preferred "view" can be determined by instructions at the time of learning and may not be directly experienced (see also Shelton \& McNamara, 2001b, 2004a, 2004b). Wang and Spelke's 
model does not seem to have sufficient internal structure to accommodate such findings.

The present experiment does not by itself rule out several alternative accounts of the findings. Scene recognition may access the same spatial representation as JRD, but in a manner that reveals effects of learned viewpoint. For example, it is possible that enduring allocentric representations were created at each learning view, but that the representation formed at the aligned view was stronger than the representation formed at the misaligned view. Scene recognition may be a more sensitive measure than $\mathrm{JRD}$, and thus able to access the weaker representation. It is also possible that enduring spatial memories are purely egocentric (i.e., viewer-centered), or even orientation free, and the setting or task demands are responsible for the pattern of results produced. The problem for such accounts is to explain the entire body of findings that have been obtained using similar methods. For example, a pure egocentric model would have difficulty accounting for the sawtooth pattern commonly found in judgments of relative direction and the numerous demonstrations that the preferred heading is determined by instructions and by properties of the surrounding environment (e.g., Mou \& McNamara, 2002; Shelton \& McNamara, 2001a, 2001b, 2004a, 2004b).

In conclusion, the present findings indicate that recognizing a place and locating a goal relative to a given position rely on different spatial representations. Recognizing one's location may rely on multiple egocentric representations. Whereas, determining the location of a goal in relation to one's current position appears to rely on a single representation of the intrinsic structure of the environment.

\section{AUTHOR NOTE}

Preparation of this article and the research reported in it were supported in part by National Institute of Mental Health Grant R01-MH57868. Correspondence concerning this article should be addressed to T. P. McNamara, Department of Psychology, Vanderbilt University, 111 21st Avenue South, Nashville, TN 37203 (e-mail: t.mcnamara@ vanderbilt.edu).

\section{REFERENCES}

Burgess, N. (2002). The hippocampus, space, and viewpoints in episodic memory. Quarterly Journal of Experimental Psychology, 55A, $1057-1080$

EASTON, R. D., \& SHOLL, M. J. (1995). Object-array structure, frames of reference, and retrieval of spatial knowledge. Journal of Experimental Psychology: Learning, Memory, \& Cognition, 21, 483-500.

Epstein, R., Graham, K. S., \& Downing, P. E. (2003). Viewpointspecific scene representations in human parahippocampal cortex. Neuron, 37, 865-876.

King, J. A., Burgess, N., Hartley, T., Vargha-Khadem, F., \& O'KeEFe, J. (2002). Human hippocampus and viewpoint dependence in spatial memory. Hippocampus, 12, 811-820.

Klatzky, R. L., LoOmis, J. M., Beall, A. C., Chance, S. S., \& Golledge, R. G. (1998). Spatial updating of self-position and orientation during real, imagined, and virtual locomotion. Psychological Science, 9, 293-298.

MaYes, A. R., Holdstock, J. S., Isaac, C. L., Montaldi, D., Grigor, J., Gummer, A., ET AL. (2004). Associative recognition in a patient with selective hippocampal lesions and relatively normal item recognition. Hippocampus, 14, 763-784.

McNamaRA, T. P. (2003). How are the locations of objects in the envi- ronment represented in memory? In C. Freska, W. Brauer, C. Habel, \& K. F. Wender (Eds.), Spatial cognition III: Routes and spatial reasoning (pp. 174-191). Berlin: Springer.

McNamara, T. P., Rump, B., \& Werner, S. (2003). Egocentric and geocentric frames of reference in memory of large-scale space. Psychonomic Bulletin \& Review, 10, 589-595.

MONTEllo, D. R., \& PiCK, H. L. (1993). Integrating knowledge of vertically aligned large-scale spaces. Environment \& Behavior, 25, 457-484.

MoU, W., \& McNamara, T. P. (2002). Intrinsic frames of reference in spatial memory. Journal of Experimental Psychology: Learning, Memory, \& Cognition, 28, 162-170.

Mou, W., McNamara, T. P., Valiquette, C. M., \& Rump, B. (2004). Allocentric and egocentric updating of spatial memories. Journal of Experimental Psychology: Learning, Memory, \& Cognition, 30, $142-157$.

Parslow, D. M., Rose, D., Brooks, B., Fleminger, S., Gray, J. A., Giampietro, V., ET AL. (2004). Allocentric spatial memory activation of the hippocampal formation measured with fMRI. Neuropsychology, 18, 450-461.

ShELTON, A. L., \& McNamara, T. P. (1997). Multiple views of spatial memory. Psychonomic Bulletin \& Review, 4, 102-106.

Shelton, A. L., \& McNamara, T. P. (2001a). Systems of spatial reference in human memory. Cognitive Psychology, 43, 274-310.

Shelton, A. L., \& McNamara, T. P. (2001b). Visual memories from nonvisual experiences. Psychological Science, 12, 343-347.

Shelton, A. L., \& McNamara, T. P. (2004a). Orientation and perspective dependence in route and survey learning. Journal of Experimental Psychology: Learning, Memory, \& Cognition, 30, 158-170.

SHELTON, A. L., \& MCNAMARA, T. P. (2004b). Spatial memory and perspective taking. Memory \& Cognition, 32, 416-426.

Sholl, M. J. (2001). The role of a self-reference system in spatial navigation. In D. R. Montello (Ed.), Spatial information theory: Foundations of geographic information science (international conference, COSIT 2001 proceedings) (pp. 217-232). Berlin: Springer.

Sholl, M. J., \& Nolin, T. L. (1997). Orientation specificity in representations of place. Journal of Experimental Psychology: Learning, Memory, \& Cognition, 23, 1494-1507.

Valiquette, C. M., McNamara, T. P., \& Smith, K. (2003). Locomotion, incidental learning, and the selection of spatial reference systems. Memory \& Cognition, 31, 479-489.

WANG, R. F., \& SPELKE, E. S. (2000). Updating egocentric representations in human navigation. Cognition, 77, 215-250.

WANG, R. F., \& SPElKe, E. S. (2002). Human spatial representation: Insights form animals. Trends in Cognitive Sciences, 6, 376-382.

Werner, S., \& Schmidt, K. (1999). Environmental reference systems for large scale spaces. Spatial Cognition \& Computation, 1, 447-473.

\section{NOTES}

1. The theories discussed subsequently also assume that memory for small-scale spaces (e.g., a classroom) is comparable to memory for large-scale spaces (e.g., a campus). Studies by McNamara, Rump, and Werner (2003) and Werner and Schmidt (1999) support this assumption (but see Montello \& Pick, 1993).

2. In addition to enduring spatial relations, each of these theories also contains an egocentric system, which computes the transient self-toobject spatial relations needed for locomotion, reaching, and grasping.

3 . Because the effect of pictured heading in scene recognition was relatively small, we conducted a replication $(N=48)$ using similar procedures except that only the scene recognition task was included and the distractors in scene recognition were mirror images of the target scenes, thus making the discrimination more difficult. Performance for $0^{\circ}$ and $135^{\circ}$ again did not differ significantly, and the average difference between familiar and novel headings was $500 \mathrm{msec}$ (cf. $200 \mathrm{msec}$ in the present experiment).

(Manuscript received April 27, 2006; revision accepted for publication August 22, 2006.) 